\title{
Energy-Efficient Cluster Reconfiguration with Fair Cluster Formations in Sensor Networks
}

\author{
Hyang-tack Lee ${ }^{1}$, Yong-hyun $\mathrm{Jo}^{2}$, Byeong-hee Roh ${ }^{1}$, and S.W. Yoo ${ }^{1}$ \\ 1 Graduate School of Information and Communication, Ajou University, \\ San 5 Wonchon-dong, Yeongtong-Gu, Suwon, 443-749, Korea \\ \{hlee, bhroh, swyoo\}@ajou.ac.kr \\ 2 National Computerization Agency, \\ NCA building, 77, Mugyo-Dong, Jung-Gu, Seoul, Korea \\ jyh@nca.or.kr
}

\begin{abstract}
In cluster-based schemes such as LEACH, cluster reconfiguration algorithm is one of the most critical issues to achieve longer lifetime of sensor networks. In this paper, we propose a new energy efficient cluster reconfiguration algorithm, called EECRA. EECRA does not require any location or energy information of sensors, and can configure clusters with fair cluster regions such that all the sensors in a sensor network can utilize their energies equally. The performances of EECRA have been compared with LEACH and LEACH-C. We also show that EECRA can be well applied to the environments that sensors are moving.
\end{abstract}

\section{Introduction}

In microsensor networks, nodes are typically constrained in energy and bandwidth. Therefore, energy-efficient design of the network is one of the most important issues to be solved. There have been much of works related to energy aware routing for sensor networks 1, 2] 3. Among those, as distributed cluster-based routing schemes, LEACH (Low-Energy Adaptive Clustering Hierarchy) has been proposed [4]. In LEACH, cluster heads play a key role to aggregate data from sensor nodes in their local cluster regions, and then to deliver the aggregate data to BS. Those cluster heads are periodically elected to prevent a specific sensor node from consuming its residual energy rapidly. However, the cluster heads are elected in distributed and probabilistic way, there exist the possibilities of poor cluster formations, in which cluster heads are located very close to each other. To overcome the problem, LEACH-C (LEACH-Centralized) [5] has been proposed. In LEACH-C, BS(base station) can determine optimum cluster formations based on the information for the locations and residual energies of all sensor nodes. LEACH-C is more effective than LEACH, but it consumes much energy compared with LEACH because all nodes have to communicate with BS at each round and it requires additional overhead for each node to know its location information through an additional communication technique such as GPS.

In this paper, we propose a new energy-efficient cluster reconfiguration algorithm, shortly called EECRA. EECRA uses distributed and probabilistic cluster 
formation method as similar as in LEACH. However, EECRA can improve the energy efficiency by preventing the possibility of poor cluster formation as in LEACH. EECRA can produce clusters with fair cluster regions such that all the sensors in a sensor network can utilize their energies equally. The cluster formations configured by EECRA at every round are as optimal as in LEACHC. Unlike LEACH-C, however, EECRA does not require any location or energy information of each sensor node. Simulation results show that EECRA outperforms both LEACH and LEACH-C.

The rest of the paper is organized as follows. In Section 2, some background on LEACH algorithm and its generic problem is explained. Then, our proposed scheme, EECRA, is illustrated in Section 3. In Section 4, some experimental results will be given. Finally, we conclude the paper in Section 5 .

\section{Background}

LEACH is a self-organizing, adaptive clustering protocol that uses randomization to distribute the energy load evenly among the nodes in microsensor network [5]. The basic operation of LEACH is as follows. In LEACH, timeline is divided into rounds as shown in Fig. 1. Each round consists of Set-up Phase and Steady-state Phase. Clusters are reconfigured in Set-up Phase, while actual data transmission can be done from nodes to the cluster head, and then to the BS in Steady-state Phase. Set-up Phase consists of three sub-phases such as Advertisement, Cluster Set-up and Schedule Creation Phases. In Advertisement Phase, each node decides whether it can be elected as a cluster head or not. Then, in Cluster Setup Phase, all nodes except for cluster heads choose their cluster head, and then cluster reconfiguration is finished. Finally, TDMA schedule for data transmission in the network is arranged in Schedule Creation Phase.

The cluster head election starts at the beginning of each round, especially in Advertisement Phase. Initially, each sensor node chooses a number between 0 and 1 randomly. If the chosen random number by $n$-th sensor node is less than a threshold value $T(n)$ as written in Eq.(11), the node elects itself to a cluster head for the corresponding round.

$$
T(n)=\left\{\begin{array}{cl}
\frac{P}{1-P \cdot\left(r \bmod \frac{1}{P}\right)} & \text { if } n \in G \\
0 & \text { otherwise }
\end{array}\right.
$$

where $P$ is the desired percentage of the cluster heads, $r$ is the current round, and $G$ is the set of nodes that have not been cluster head in the last $1 / P$

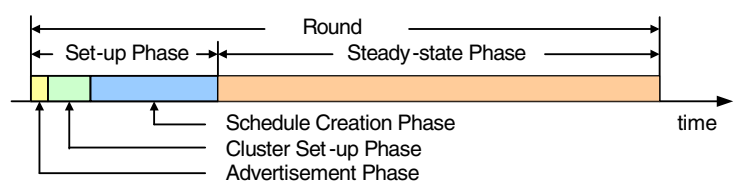

Fig. 1. Timeline of LEACH operation 


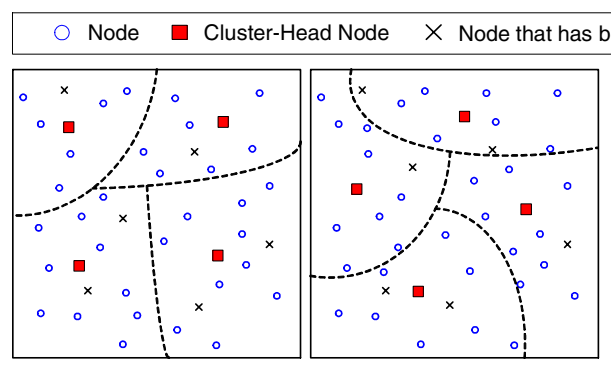

(a)

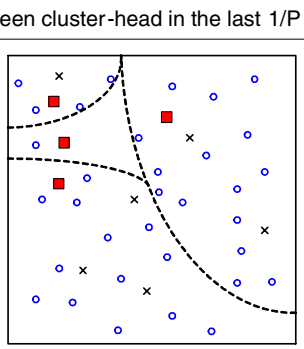

(b)

Fig. 2. Possible example of cluster formations: (a) good case (b) poor case

rounds. According to Eq.(1), LEACH ensures that all nodes become a cluster head exactly once during consecutive $1 / P$ rounds.

Because cluster heads are elected in only probabilistic way as shown in Eq.(1), there is no way to consider the formation of clusters in LEACH. Thus, there exists some possibility of both good and poor cluster formations as shown in Fig. 2 In good cluster formations as in Fig. 2(a), all sensor nodes can consume their energy evenly in average. On the other hand, in some poor cluster formations as shown in Fig. 2(b), in which adjacent nodes can be elected as cluster heads, sensor nodes with longer distances to corresponding cluster head consume much more energies than those with shorter distances. In addition, collisions can be occurred frequently in the network due to short distance between cluster heads.

To overcome the problem of poor cluster formation in LEACH, LEACH-C has been proposed $[5$. As mentioned in Section 1, however, LEACH-C has also problems of overheads for each node to know its location and deliver its location and energy information to BS. The overhead results in consuming much energy compared with LEACH.

\section{Energy Efficient Cluster Reconfiguration Algorithm}

\subsection{Basic Operation of EECRA}

Fig. 3 shows the basic timeline of the proposed EECRA operation, in which timelines are divided into rounds as in $\mathrm{LEACH}$. Unlike LEACH, the Advertisement Phase of EECRA consists of $k$ stages where $k$ denotes the predefined number of cluster heads in the network. There exists only one stage in the Advertisement Phase in LEACH. This means that there are $k$ chances for each node to become a cluster head in EECRA, while only one in LEACH. Let $N$ be the total number of sensor nodes, and $T(n, s)$ be the threshold value at $s$-th stage that $n$-th sensor node can become a cluster head, where $s=0,1, \ldots, k-1$. Then, we have

$$
T(n, s)=\left\{\begin{array}{cc}
\frac{1}{\left\{N-k \cdot\left(r \bmod \frac{N}{k}\right)\right\} \cdot\left(1-\frac{s}{k}\right)} & \text { if } n \in G \\
0 & \text { otherwise }
\end{array}\right.
$$




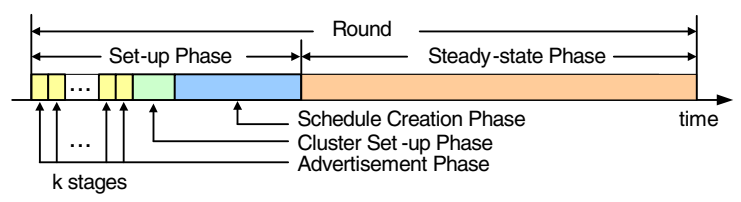

Fig. 3. Timeline of EECRA operation

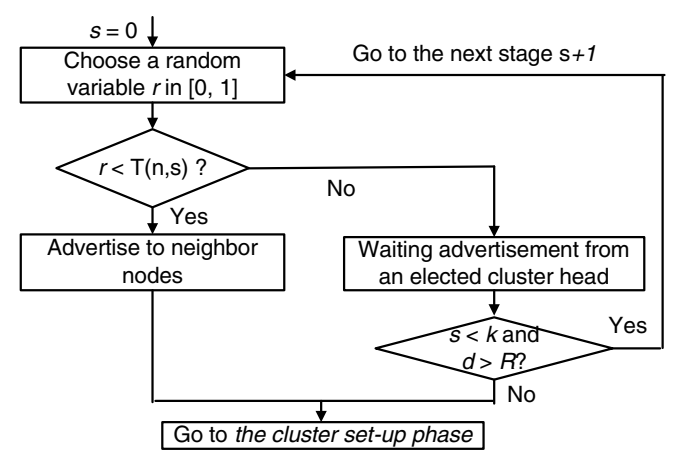

Fig. 4. Cluster head election process of $n$-th node

where $r$ is the current round number. The derivation of Eq.(2) is illustrated in Appendix.

The cluster head election process at each stage is shown in Fig. 4. At the beginning of stage $s$, sensor nodes eligible to become a cluster head select a value between 0 and 1 randomly. If the selected random number by a node is less than the threshold $T(n, s)$, the node is elected as a cluster head for the corresponding stage. The node elected as a cluster head broadcasts its advertisement message to neighbor nodes within a certain range. Then, those neighbor nodes receiving the advertisement message estimate the distance $d$ from itself to the cluster head by considering the received message signal power. The nodes with the estimated distance less than $R$ do not take part in the cluster head election process at the next stage $s+1$. We will discuss the value of $R$ in next subsection. In other words, at the next stage $s+1$, only rest nodes that are not belong to the ranges covered by the cluster heads elected at the previous stages can participate in the cluster head election process.

Likewise, EECRA prevents the possibility of unsuitable cluster formations. To do so, EECRA has a little longer Set-up Phase duration than LEACH since it has $k$ stages. However, we will show in experimental results this can provide more energy-efficient operation of sensor networks than LEACH.

\subsection{Analysis of $R$ in EECRA}

In EECRA, a cluster head elected at each stage broadcast its advertisement message to neighbor nodes within the circle range with radius $R$, then nodes 
within the range do not take part in head election process at the next stage during corresponding round. Accordingly, to find out suitable value of $R$ is very important to configure efficient cluster formations. In this paper, we calculate the $R$ value considering the area of sensor networks.

Consider a sensor network with $M \times M$ size. Let us assume an ideal situation that sensor nodes are uniformly distributed on the sensor network and $k$ elected cluster heads are evenly arranged. Then, we have the following approximation

$$
M^{2}=k \pi R_{O}^{2}
$$

where $R_{O}$ is the radius for the advertisement region covered by each cluster heads in the above ideal situation. Then, we have $R_{O}=\frac{M}{\sqrt{\pi k}}$. The distance $R_{O}$ is suitable only for the ideal situation when the cluster heads are evenly located such that the sum of areas covered by each cluster head is as similar as the area of entire sensor network. However, when some of cluster heads are located on the region near to edge of the network, it becomes unsuitable because the advertisement regions covered by those clusters are smaller than $M^{2} / k$, and it does not satisfy the optimal condition of $R_{O}$ as in Eq.(3). To increase the number of nodes that do not need to participate in cluster election process at rest stages and to avoid the poor cluster formation, we can consider some larger values than $R_{O}$. However, we can intuitively expect that the larger the value of $R_{O}$, the higher possibility that cluster nodes at border area can be elected as cluster heads. Since this is not desirable, it needs a certain upper limit of the increase of the radius. Let assume an extreme case when $k=1$ and the cluster head is elected at the borders of the sensor network. Then, with the radius of $2 R_{O}$, the cluster heads can cover the whole sensor network range. So, we can get the upper bound of the radius $2 R_{O}$. On the other hand, as the number of cluster heads $(k)$ increases, especially let assume that it approaches to infinite, even small area covered by each cluster heads with corresponding small Ro is enough to satisfy the ideal situation of Eq.(3). Likewise, the radius $R$ is highly related to the area of sensor networks as well as the number of clusters.

Let $R_{A}$ be the average radius of the sensor network. For sensor networks with $M \times M$ size, it can be approximated by $M^{2}=\pi R_{A}{ }^{2}$. From the intuition that we discussed above, if we define the radius $R \equiv\left(1+\frac{R_{O}}{R_{A}}\right) R_{O}$, then we have

$$
R=\left(1+\frac{1}{\sqrt{k}}\right) R_{O}
$$

It is noted that the factor in front of $R_{O}$ of right side of Eq.(4) has the range between 1 and 2 . This provides the consistency with the intuitions that we used for the derivation of $R$. That is, $R=2 R_{O}$ when $k=1$, and $R \rightarrow R_{O}$ when $k \rightarrow \infty$.

\section{Experimental Result}

\subsection{Simulation Environment}

We carried out the simulations using the Network Simulator ns-2 [6] [7. For the simulation, we consider a sensor network with size of $100 \times 100$ and 100 sensor 
Table 1. Basic parameters used for the simulation

\begin{tabular}{|c|c|}
\hline Network Size $(M \times M)$ & $100 \times 100$ \\
\hline Location of the nodes & from $(0,0)$ to $(99,99)$ \\
\hline Location of Base Station & $(50,175)$ \\
\hline The number of nodes $(N)$ & 100 \\
\hline Desired number of clusters $(k)$ & 5 \\
\hline Initial Energy for each node & $2 \mathrm{~J}$ \\
\hline Spreading factor & 8 \\
\hline Changing clusters & Every 20 seconds \\
\hline
\end{tabular}

nodes arbitrarily distributed on the sensor network. In our simulation, we use the same simulation environment and radio energy model as discussed in [5]. Our simulation environment is described in Table 1. According to Eq.(4), we selected the radius $R$ for EECRA as 37 .

\subsection{Simulation Results}

In the Case of Static Nodes. In Fig. [5(a), performances of the system lifetime defined as the time until all nodes are dead are compared. The system lifetime of LEACH-C is shorter than both LEACH and EECRA. This is because in LEACH$\mathrm{C}$, each node is required to maintain its location and energy information, and has to deliver the information to BS. On the other hand, these overheads are not needed for LEACH and EECRA. So, the energy of each node in LEACH$\mathrm{C}$ is consumed faster than other schemes. And, we can also see from Fig. [5)(a) that EECRA has longer lifetime than LEACH. This indicates that EECRA can operate nodes in a very energy-efficient way. Though the system lifetime of LEACH-C is shorter than other schemes, from the viewpoints of the amount of delivered data, LEACH-C outperforms other schemes as shown in Fig. 55(b). This is because LEACH-C can configure optimal cluster formations since BS knows all the operation information of sensor nodes. The data delivery performance of EECRA shows better than that of LEACH. Since LEACH configures clusters based only on probabilistic threshold value, poor cluster formation can be made. Under poor cluster formations, nodes may consume more energy for the same amount of data delivery due to collisions and far distances between some nodes, cluster heads and BS. However, since EECRA forms clusters in consideration of their formations, it can reduce the problem due to poor cluster formations.

In Fig. 6, throughput performances are shown. Fig. 6)(a) and (b) present the total amount of data over the time and the total amount of data per given amount of energy received at BS, respectively. As we can expect, BS under LEACH-C receives more amount of data than other schemes because of the optimal cluster formation of LEACH-C with the global knowledge of the network. That is, once clusters are formed in LEACH-C, it requires less energy for data transmission between cluster heads and their cluster member nodes. EECRA shows lower throughput than LEACH-C, but much better than LEACH. This means that EECRA can produce clusters with better formations than LEACH. 


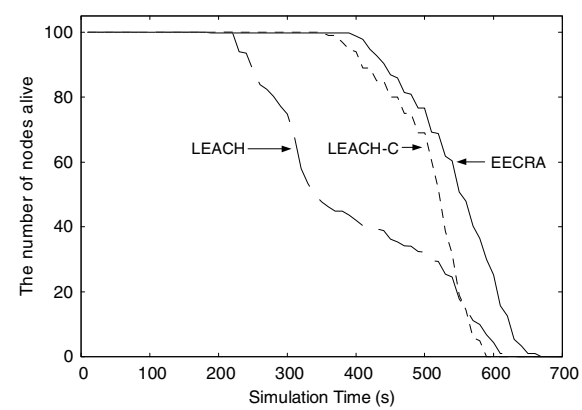

(a)

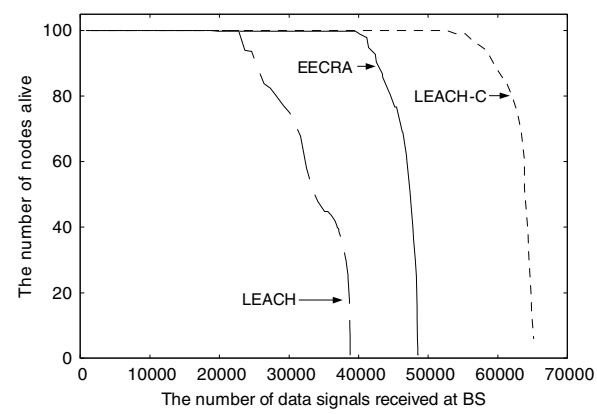

(b)

Fig. 5. Lifetime Performances. (a) number of nodes alive along time (b) number of nodes alive per the amount of data sent to BS.

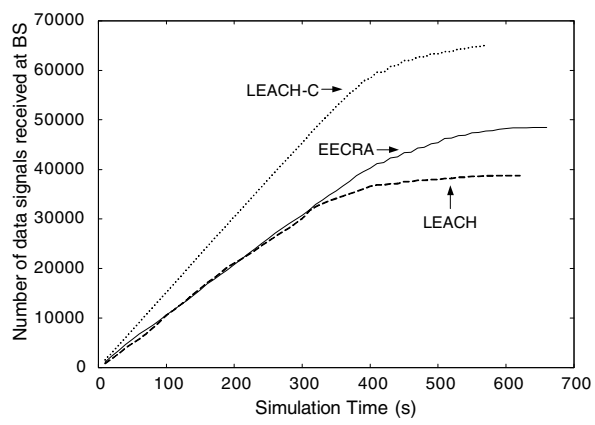

(a)

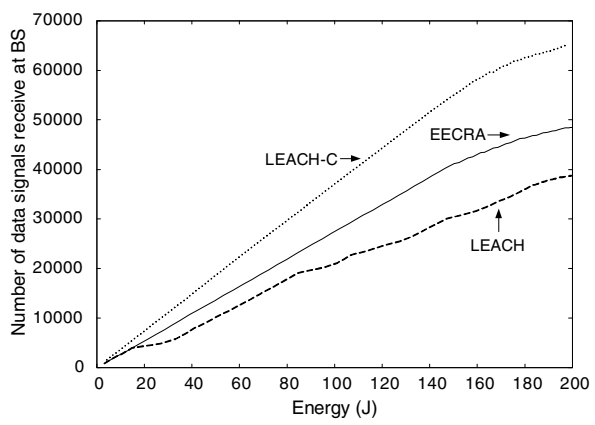

(b)

Fig. 6. Throughput performances (a) the total number of received data signals at BS over time (b) the total amount of data received at BS per given amount of energy

In the Case of Mobile Nodes. For the simulation of the environment that sensor nodes are moving, we used Random Waypoint model presented in [8]. At every second, each node randomly chooses a destination and moves toward it with a velocity uniformly chosen from the range $\left[0, V_{\max }\right]$, where $V_{\max }$ is the maximum allowable velocity for every mobile node[9]. The parameters for Random Waypoint model are described in Table 2. We set the values of $V_{\max }$ vary between 1 and $7 \mathrm{~m} / \mathrm{s}$ to show the efficiency of the network in various mobile environment. In Fig. 7(a), it shows the system lifetime of EECRA over the time for several velocities. As the velocity increases, the number of nodes alive is decreased rapidly along time. We investigated that for other schemes such as LEACH and LEACH-C also show similar pattern as in EECRA of Fig. 77(a). In Fig. [7(b), the lifetimes for EECRA, LEACH and LEACH-C are compared. We can see that EECRA keeps the network longer than LEACH and LEACH-C. And, as in the static node cases, LEACH-C's lifetimes are shorter than LEACH. We can explain the reason of these phenomena as in the static node cases. 
Table 2. Parameters for Random Waypoint model

\begin{tabular}{|c|c|}
\hline$V_{\max }$ & varies between 1 and $7 \mathrm{~m} / \mathrm{s}$ \\
\hline Moving distance per second & {$\left[0 \sim V_{\text {max }}\right] \mathrm{m}$} \\
\hline Direction & {$[0 \sim 2]$ Radian } \\
\hline Moving distance per second at x-axis & {$\left[0 \sim V_{\max }\right] \cos ([0 \sim 2]$ Radian $) \mathrm{m}$} \\
\hline Moving distance per second at y-axis & {$\left[0 \sim V_{\max }\right] \sin ([0 \sim 2]$ Radian $) \mathrm{m}$} \\
\hline
\end{tabular}

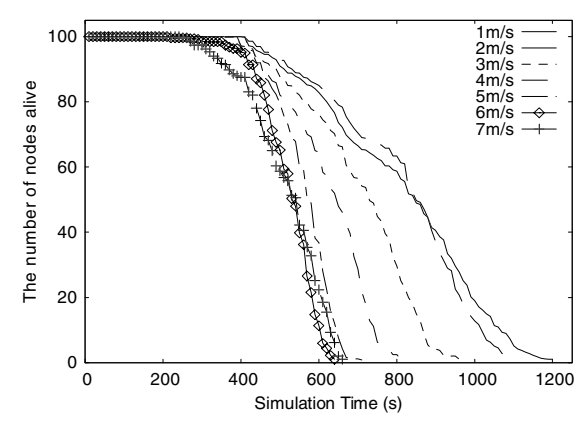

(a)

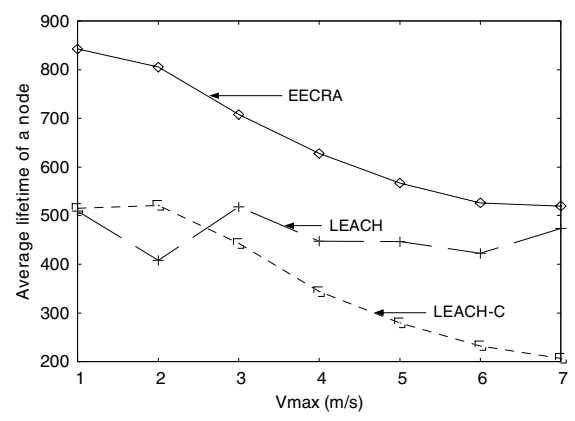

(b)

Fig. 7. System lifetime in mobile environments (a) EECRA over time with various velocities (b) comparisons of average node lifetime for three schemes

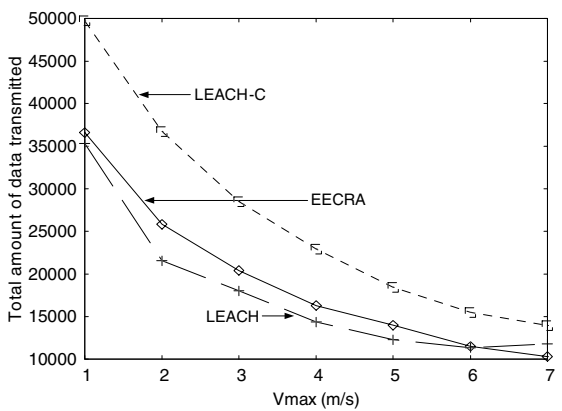

(a)

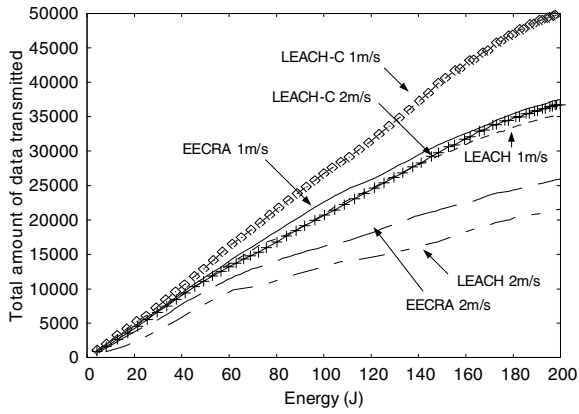

(b)

Fig. 8. Throughput in mobile environments (a) total amount of transmitted data (b) total amount of data transmitted per given amount of energy

Fig. 8(a) compares the total number of data signals transmitted during the lifetimes of EECRA, LEACH and LEACH-C. As the velocity increases, the amount of delivered data signals tends to decrease. For all velocities, as we can easily expect, LEACH-C sends more data than EECRA and LEACH. And, EECRA shows always better performance than LEACH. In Fig. 8(b), we show the total amount of data signals transmitted per given amount of energy for those three schemes. LEACH-C always shows the best performances. At $1 \mathrm{~m} / \mathrm{s}$ 
velocity, EECRA shows slightly better performances than LEACH. However, at $2 \mathrm{~m} / \mathrm{s}$ velocity, we can see much more performance differences between EECRA and LEACH. Likewise, as velocity increases, EECRA can achieve better performances than LEACH.

Our simulation results show that the system lifetime performance of EECRA outperforms that of other schemes such as LEACH and LEACH-C. However, for the data throughput, LEACH-C shows the best performances, and EECRA is better than LEACH. Though LEACH-C provides the best throughput performances, it has some limitations such as much shorter system lifetime and more complexities to keep the location and energy information in both sensor nodes and BS. From the operational complexity's viewpoints, EECRA can be operated with lower complexity as similar as LEACH, but EECRA provides better performances in both lifetime and throughput than LEACH. In these points of views, EECRA can be more effective than LEACH-C.

\section{Conclusion}

In this paper, we proposed a new energy-efficient cluster reconfiguration algorithm called EECRA. EECRA can reduce the possibility of poor cluster formations appeared in LEACH. With the property, we showed that EECRA can achieve improved lifetime performances than LEACH and LEACH-C under both static and mobile nodes environments. And, EECRA provides reasonable data delivery throughput better than LEACH, but less than LEACH-C. However, EECRA can be implemented less complexity than LEACH-C.

From the performance results of EECRA obtained from experiments, we can conclude that EECRA can be well applied to the situation where longer system operation is required and medium amount of sensing data is requested to be exchanged continuously.

\section{Appendix: Derivation of Equation (2)}

For the derivation of Eq.(2), we let the time at round $r$ and stage $s$ be $(r, s)$ where $r=0,1,2, \ldots$ and $s=0,1, \ldots, k-1$, and define the following terms at $(r, s)$.

$P_{n}(r, s) \quad$ probability that node $n$ become a cluster head $(n=1, \ldots, N)$

$C(r, s) \quad$ number of nodes that can take part in cluster head process

$E_{C H}(r, s)$ expected number of nodes that can be elected as cluster heads

From the definition, $C(r, 0)$ is the number of nodes that can be candidates of cluster heads at the beginning of round $r$. Then, $C(r, 0)=N-k \cdot\left(r \bmod \frac{N}{k}\right)$. In EECRA, it is noted that nodes within the ranges with radius $R$ from cluster heads elected at every stages can not take part in the cluster head process at the next stage. The average number of nodes belongs to the range covered by each cluster head can be $C(r, 0) / k$. Accordingly, we can write

$$
C(r, s)=C(r, 0) \cdot\left(1-\frac{s}{k}\right)
$$


Ideally, one cluster head is elected at each stage. So, the probability $P_{n}(r, s)$ satisfies the following condition.

$$
E_{C H}(r, s)=\sum_{n=1}^{N} P_{n}(r, s)=1
$$

Let $G$ be the set of nodes that have not been elected as cluster heads in the last $N / k$ rounds, and $P_{n \in G}(r, s)$ be the probability that an arbitrary node $n(n \in G)$ becomes a cluster head at time $(r, s)$. The nodes that have not been cluster head have identical $P_{n}(\cdot)$ at each stage. Then, we can rewrite Eq. (A2) as following

$$
E_{C H}(r, s)=C(r, s) \cdot P_{n \in G}(r, s)=1
$$

From Eq.A3 , we have

$$
P_{n \in G}(r, s)=\frac{1}{C(r, s)}
$$

It is noted that Eq. (A4) is the probability that only one node can be elected as the cluster head at a certain stage. Substituting Eq.(A1) into Eq. (A4), we have the equation as same as Eq.(2).

Acknowledgements. This work was supported by grant (No. 05A3-I3-10) from Ubiquitous Autonomic Computing and Network Project sponsored by the Ministry of Information and Communication, Korea.

\section{References}

1. Edger H. Callaway, Wireless Sensor Networks: Architectures and Protocols, Auerbach Publications, August 2003

2. Sameer Tilak, Nael B. Abu-Ghazaleh, and Wendi Heinzelman, "A Taxonomy of Wireless Micro-Sensor Network Models," ACM Mobile Computing and Communications Review, Vol. 6, No. 2, pp. 28-36, April 2002

3. I.F.Akyildiz, W.Su, Y.Sankarasubramaniam, E.Cayirci, "A survey on Sensor Networks," IEEE Communications Magazine, Vol. 40, No. 8, pp. 102-114, August 2002

4. W. Heinzelman, A. Chandrakasan, and H. Balakrishnan, "Energy-Efficient Communication Protocol for Wireless Microsensor Networks," Proceeding of Hawaii Conference on System Sciences, January 2000

5. W. Heinzelman, A. Chandrakasan, and H. Balakrishnan, "An Application-Specific Proto-col Architecture for Wireless Microsensor Networks," IEEE Transactions on Wireless Communications, Vol. 1, No. 4, October 2002

6. UCB/LBNL/VINT, "Network Simulator ns-2," http://wwwmash.cs.berkeley.edu/ns.

7. W.Heinzelman, A.Chandrakasan, and H.Balakrishnan, uAMPS ns Code Extensions, http://wwwmtl.mit.edu/ research/icsystems/uamps/leach

8. F.Bai, N.Sadagopan, A.Helmy, "The IMPORTANT Framework for Analyzing the impact of Mobility on Performance of Routing for Ad Hoc Networks," Ad-Hoc Networks, Vol. 1, No. 5, pp. 383 - 404, November 2003

9. L. Breslau, D. Estrin, K. Fall, S. Floyd, J. Heidemann, A. Helmy, P. Huang, S. McCanne, K. Varadhan, Y. Xu, H. Yu, "Advances in network simulation," IEEE Computer Magazine, Vol. 33, No. 5, pp. 59-67, May 2000 